\title{
Correlation between Single Molecular Dynamics and Biological Functions of Antimicrobial Peptide Melittin
}

Cheng Xu, ${ }^{\dagger, \S}$ Wendong Ma, ${ }^{\dagger, \S}$ Kang Wang, ${ }^{\dagger}$ Kejie He, ${ }^{\dagger}$ Zhonglan Chen, ${ }^{\dagger}$ Jiaojiao Liu,${ }^{\dagger}$ Kai Yang, ${ }^{,}{ }^{, \dagger}$ and Bing Yuan ${ }^{*}+\dot{T}$

${ }^{\dagger}$ Center for Soft Condensed Matter Physics and Interdisciplinary Research \& School of Physical Science and Technology, Soochow University, Suzhou 215006, P. R. China

tCollege of Physics and Electronic Engineering \& Jiangsu Laboratory of Advanced Functional Materials, Changshu Institute of Technology, Changshu 215500, P. R. China

${ }^{\S}$ C.X. and W.M. contributed equally to this work.

\section{Corresponding Authors}

*E-mail: yangkai@ suda.edu.cn. Phone: +86-512-65220239.

*E-mail: yuanbing@suda.edu.cn. Phone: +86-512-65220239.

Supplementary images and corresponding notes (Figure S1-S10)

Supplementary tables (Table S1-S3)

Data analysis details

Supplementary equations and corresponding notes

Experimental details and corresponding notes 


\section{Supplementary images and corresponding notes}
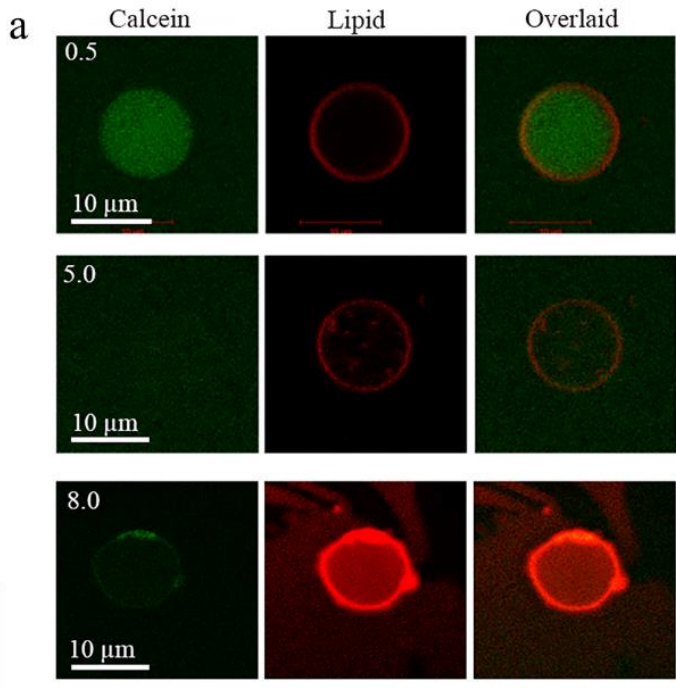

d
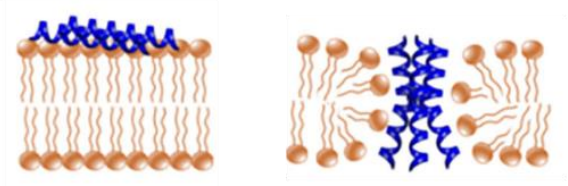

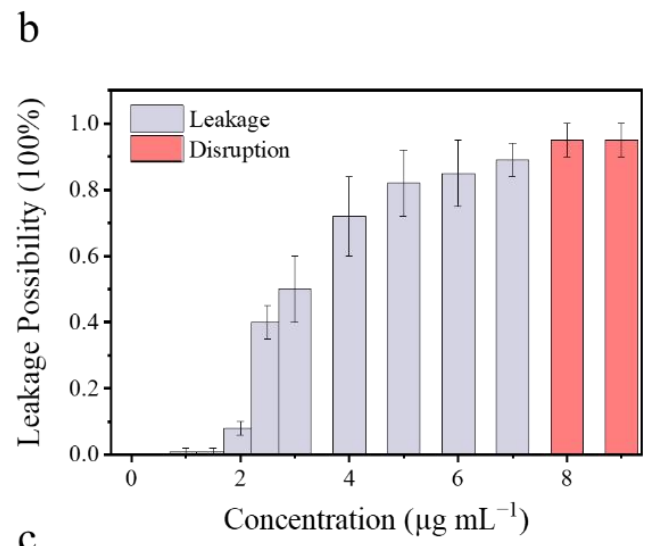

c

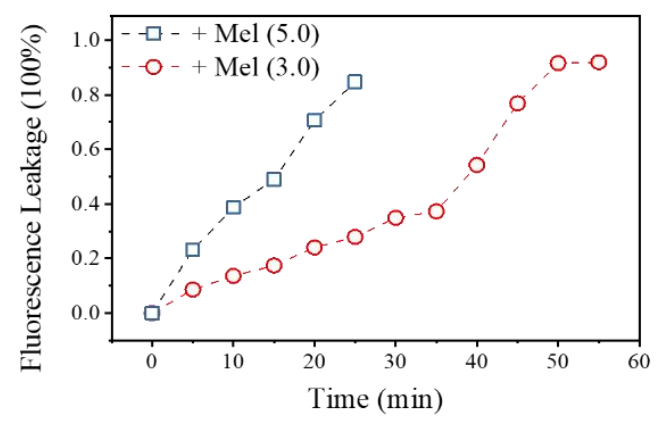

Figure S1. Concentration- and time-dependent dye leakage from the calcein-encapsulated GUVs due to melittin exposure. (a, b) Mel-concentration dependence of the GUV leakage. (a) Representative confocal images showing the GUVs exposed to melittin at different concentrations of $0.5,5.0$, or $8.0 \mu \mathrm{g} \mathrm{mL}^{-1}$. Images were taken in the green (calcein) and red (lipid) channels, respectively. At $8.0 \mu \mathrm{g} \mathrm{mL}{ }^{-1}$, most of the GUVs have been disrupted, with lipid molecules deposited on the substrate shown as red background in the image. (b) Number percentage of the leaked GUVs under observation due to melittin exposure at different concentrations. The values are acquired 15 min after melittin addition into the solution. (c) Typical time-evolution of the fluorescence leakage from a GUV due to melittin exposure at 3.0 or $5.0 \mu \mathrm{g} \mathrm{mL} L^{-1}$. (d) Cartoons show the surfacial adsorption and transmembrane insertion states of peptides.

\section{Corresponding notes}

The calcein-encapsulated GUVs kept stable for more than four hours under observation. The addition of melittin at low concentrations did not induce distinguishable disturbance to the vesicles either, until the concentration increased to a threshold value of $\sim 2.5 \mu \mathrm{g} \mathrm{mL}{ }^{-1}$. At this concentration, 
calcein leakage began to occur on 30 40\% of the vesicles under observation (observed 15 min after melittin addition). At $5.0 \mu \mathrm{g} \mathrm{mL} \mathrm{m}^{-1}$, this percentage was increased to $75 \sim 85 \%$. Moreover, at an even higher concentration of $8.0 \mu \mathrm{g} \mathrm{mL}-1$, GUV rupture occurred immediately upon melittin addition, indicating a drastic membrane disruption effect of peptides then. The peptide-concentration dependence of the GUV leakage behavior was summaried in Figure S1b. Such a peptideconcentration-dependent dye leakage indicates the inter-peptide cooperation for transmembrane insertion and poration.

Figure S1c shows time dependence of the melittin-induced transmembrane leakage of calcein, with a peptide concentration of $3.0 \mu \mathrm{g} \mathrm{mL}^{-1}$, being around the threshold leakage value, or $5.0 \mu \mathrm{g}$ $\mathrm{mL}^{-1}$, much higher than the threshold value. In comparison of the linear dependence at $5.0 \mu \mathrm{g} \mathrm{mL}^{-1}$, the time-dependent increase of leakage rate (i.e., slope of the intensity-time profile) indicates the gradual adsorption and accumulation process of peptides on membrane surface for poration.

Experimental and simulation results from our and other research groups have suggested that, melittin tends to adsorb, accumulate and aggregate on membrane surface, until reaching a critical local number density for membrane insertion and poration. ${ }^{1-5}$ Therefore, peptide concentrations of 0.5 and $5.0 \mu \mathrm{g} \mathrm{mL}^{-1}$ were adopted in our following experiments, at which the melittin molecules were supposed to be at a surface adsorption and transmembrane insertion state, respectively, referring to the two most important membrane interaction states during Mel action. 

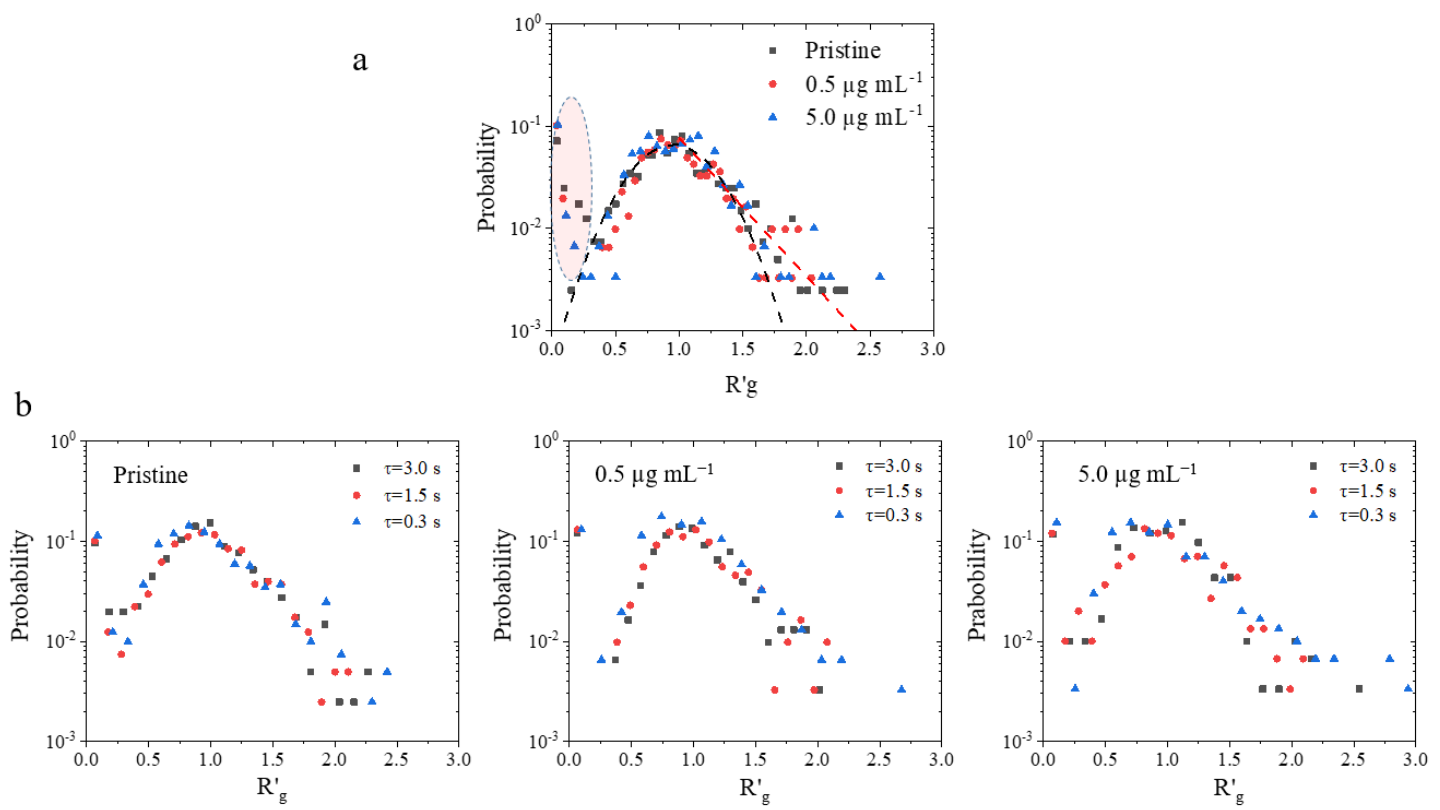

Figure S2. PDF histogram of the normalized radius of gyration $\left(R_{g}^{\prime}\right)$ of lipid trajectories. (a) The solid points in black, red and blue refer to the case of a pristine bilayer, or a bilayer with melittin at 0.5 or $5.0 \mu \mathrm{g} \mathrm{mL} \mathrm{m}^{-1}$, respectively. A Gaussian function centered at 1.0 and an exponential function as $y=1.726 \exp (-3.117 x)$ were used to fit the data points. The dashed ellipse indicates the cutoff value used to define the immobile trajectories, which are representatively shown in red in Figure 1b in the main text. (b) PDF histograms of the normalized radius of gyration $\left(R_{g}^{\prime}\right)$ of lipid trajectories in membrane without or with melittin exposure at 0.5 or $5.0 \mu \mathrm{g} \mathrm{mL} L^{-1}$, respectively. The distributions were obtained with different $\tau$ values.

\section{Notes}

To describe the displacement range of a lipid within certain time interval, the radius of gyration of the trajectory based on $R_{g}^{2}=\frac{1}{N} \sum_{i=1}^{N}\left[\left(x_{i}-\langle x\rangle\right)^{2}+\left(y_{i}-\langle y\rangle\right)^{2}\right]$ where $N$ is the total number of time steps in each trajectory, $x_{i}$ and $y_{i}$ are the projection of the position of each trajectory step on the $\mathrm{x}$ - and $\mathrm{y}$-axis, respectively, and $\langle x\rangle$ and $\langle y\rangle$ are their mean values, was calculated. To further compare the $R_{g}$ values in different cases, the normalized radius of gyration $R_{g}^{\prime}=$ $R_{g} /\left\langle R_{g}^{2}\right\rangle^{1 / 2}$, where $\left\langle R_{g}^{2}\right\rangle^{1 / 2}$ is the mean value of $R_{g}$, was used instead. Figure S2a shows the probability density function (PDF) of $R_{g}^{\prime}, h\left(R_{g}^{\prime}\right)$ at various peptide concentrations. It is found that $h\left(R_{g}^{\prime}\right)$ mainly distributes in three regions: in addition to the Gaussian peak centered at 
$R_{g}^{\prime}=1.0$, there are still scattered spots at the low $\left(R_{g}^{\prime}<0.3\right.$, marked with dashed ellipse $)$ and high (referring to many long-range moving lipids) value ranges; such distribution indicates the lipid diffusion in the systems is not an ideal Brownian motion. The spots in the low value range occupy only a tiny percentage below 10\%, which correspond to the immobile molecules (marked in red in Figure 1b). These immobile spots are not included in the following calculations of the main text unless otherwise noted. In addition, no obvious distinction is observed among these three systems or with different delay time $\tau$ (Figure S2b).

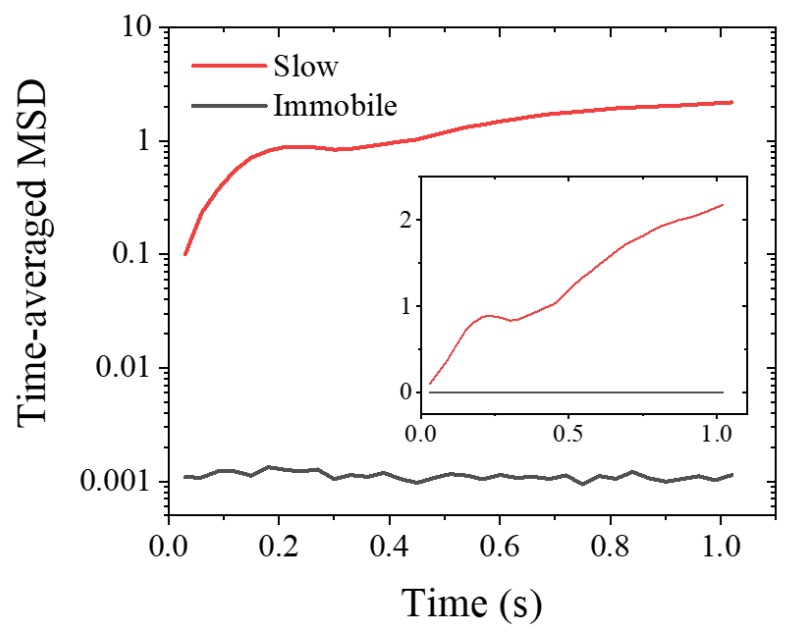

Figure S3. Time-averaged MSD of representative trajectories of the "immobile" and "slow" diffusive lipids. Inset shows the profiles in a linear coordinate.

Notes

Proportion of immobile lipids in the three systems (i.e., with melittin at $0,0.5$, or $5.0 \mu \mathrm{g} \mathrm{mL}^{-1}$ ) was statistically analyzed, to be $8.5 \%, 8.8 \%$, and $8.6 \%$, respectively. Such an independence on melittin concentration indicates that the immobility of these lipids is not induced by peptide exposure. Therefore, the immobile lipids were not included in the following calculations as we mentioned in the main text (Page 6). 


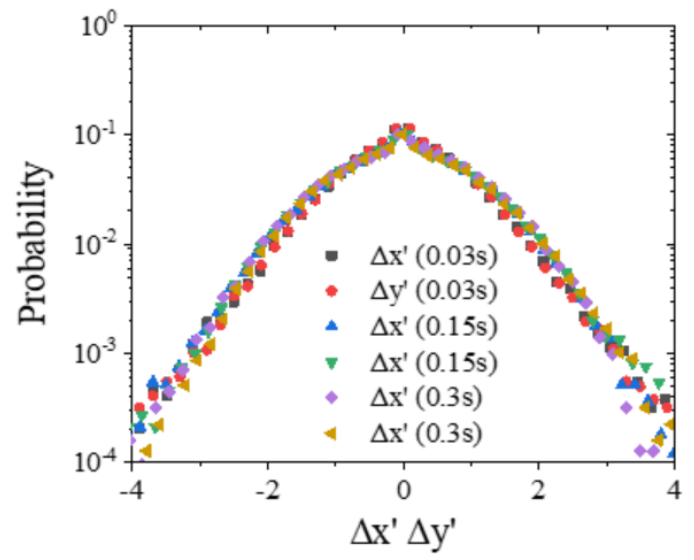

Figure S4. Normalized histogram of the displacements $\left(\Delta \mathrm{x}^{\prime}\right.$ and $\left.\Delta \mathrm{y}^{\prime}\right)$ for the trajectories of mobile lipids on a pristine membrane, calculated with different $\tau$ values.

a

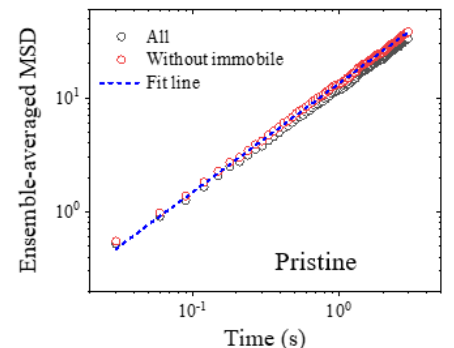

b

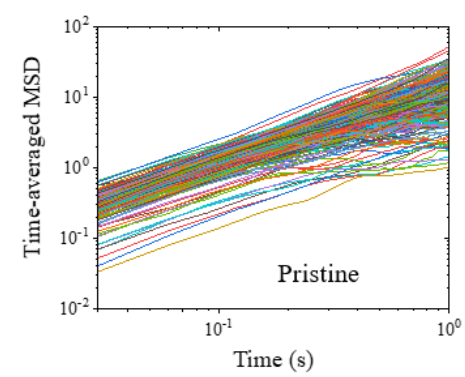

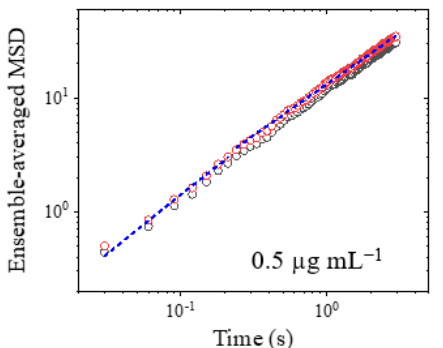
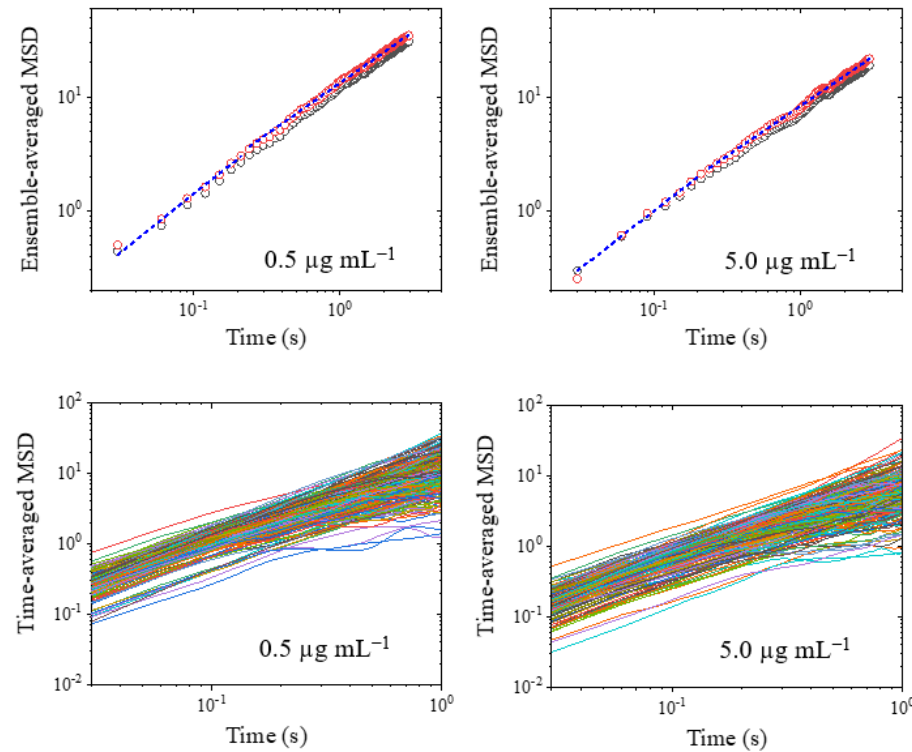

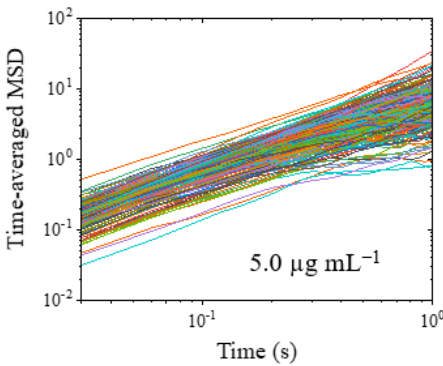

Figure S5. Influence of melittin exposure on diffusion behavior of lipids. MSD analysis of lipid trajectories on a pristine bilayer or bilayer exposed to melittin at 0.5 or $5.0 \mu \mathrm{g} \mathrm{mL}^{-1}$. (a) Ensembleaveraged MSD in each condition. The black circles are obtained when both the mobile and immobile trajectories are included in the ensemble average. The red circles are obtained when the immobile trajectories are excluded in the calculation. (b) Time-averaged MSD of an overall of 400 representative lipid trajectories in each condition. Normalized PDF histograms of the diffusion coefficient $\left(D_{L}\right)$ of these lipids are shown in Figure 1d in the main text. 

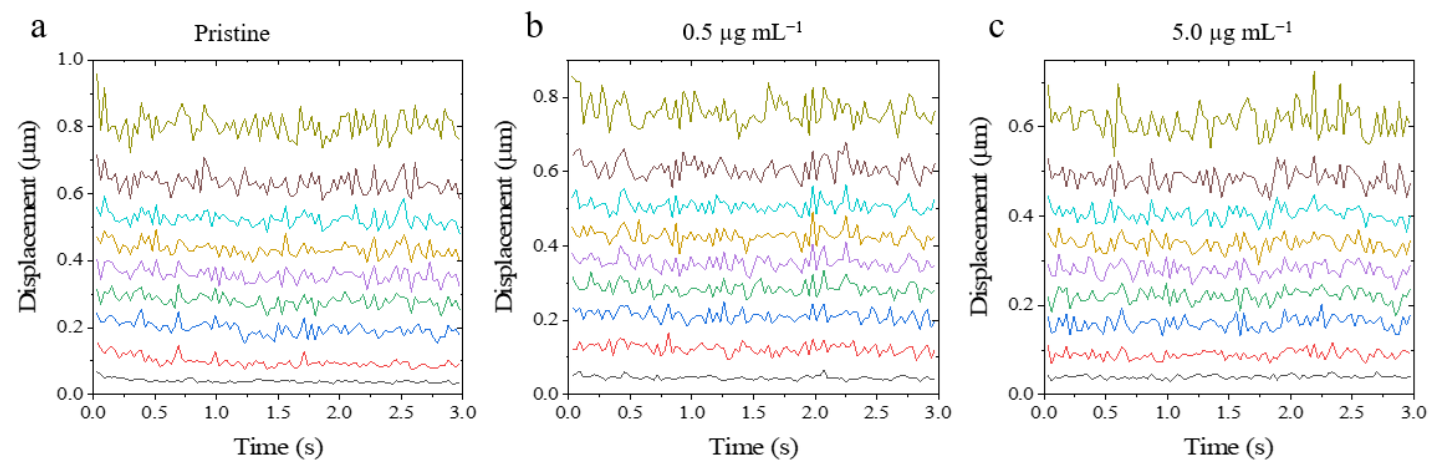

Figure S6. The 10th quantile profiles of displacements of lipids in a pristine bilayer (a) or bilayer exposed to melittin at 0.5 (b) or $5.0 \mu \mathrm{g} \mathrm{mL}^{-1}$ (c).

\section{Note}

In each system, we calculated the 10th quantile of every frame for all the trajectories. It can be figured out that the lines which link the 10th quantile of every frame keep parallel as time lapse. This indicates that the random progress is ergodic. ${ }^{6}$

$\mathrm{a}$

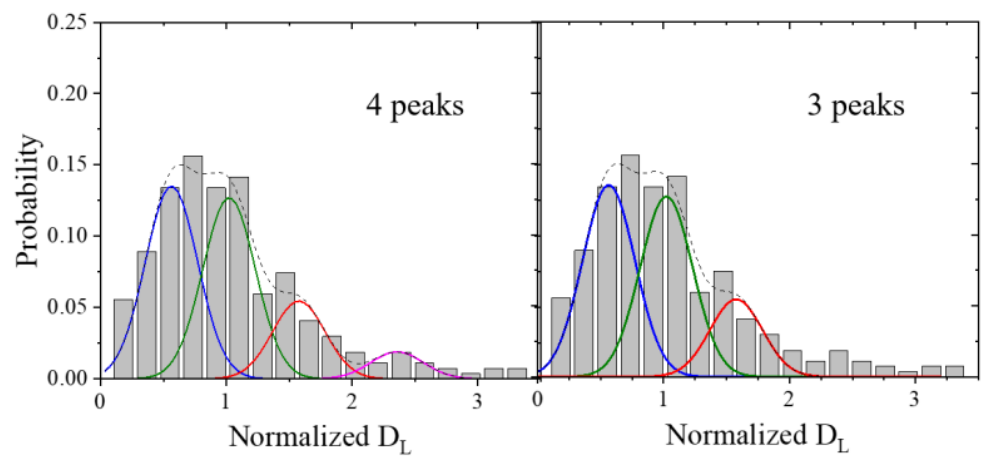

b

\begin{tabular}{|l|l|l|l|}
\hline & $\mathrm{R}^{2}$ & Fit Std Error & F-value \\
\hline 3 peaks & 0.937 & 0.017 & 27.083 \\
\hline 4 peaks & 0.951 & 0.016 & 22.050 \\
\hline
\end{tabular}

Figure S7. Multi-peaks Gaussian fittings of the PDF histogram of the diffusion coefficient $\left(D_{L}\right)$ of lipids with Mel at $0.5 \mu \mathrm{g} \mathrm{mL}^{-1}$ as shown in Figure 2d. (a) The histogram was fitted with four or three peaks. (b) Comparison of the goodness of fit tests.

\section{Notes}

For a good comparison among these three systems, we tried to locate the peaks in different 
conditions at the same position. Therefore, histogram in the left panel in Figure 1d (pristine membrane) was firstly fitted with a single Gaussian function. The peak was determined thereafter. Then, with this peak, histogram in the right panel $\left(5.0 \mu \mathrm{g} \mathrm{mL}^{-1}\right)$ was fitted with the multi-peaks Gaussian fitting, and the second peak was acquired (referring to the "slow" diffusive lipids). Finally, histogram in the middle panel $\left(0.5 \mu \mathrm{g} \mathrm{mL} L^{-1}\right)$ was fitted with multi-peaks Gaussian fittings based on these two peaks, and the third one (referring to the "fast" diffusive lipids) was obtained. Results of the goodness of fit tests were shown in Table S4.

It is found that PDF histogram of the $0.5 \mu \mathrm{g} \mathrm{mL}-1$ case can be fitted with three or four peaks (as shown in Figure S7). Results of the goodness of fit tests show no much difference between these two fittings. Moreover, the fourth peak at $\sim 2.4$ would not influence the conclusion we made in the main text but make the condition more complicated. Therefore, the 3-peak fitting result was selected and shown in the main text, for a concise comparison among the three systems.

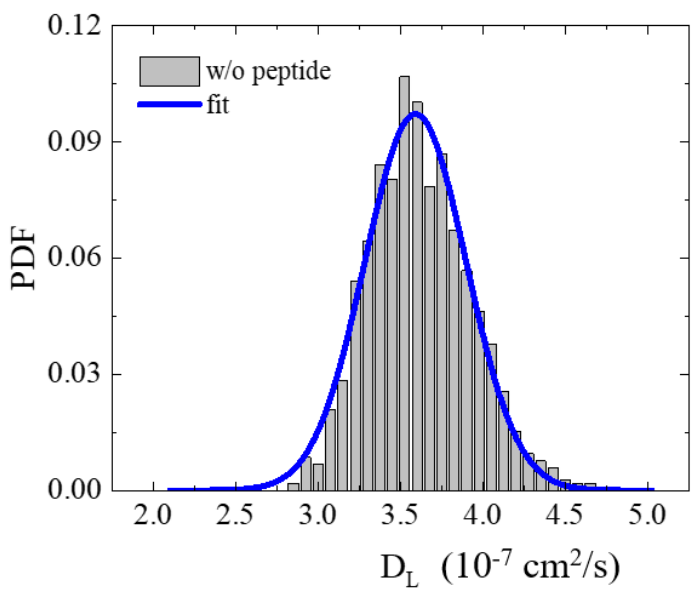

Figure S8. PDF of lipids in a pristine bilayer calculated from MD simulations. The blue curve is Gaussian fit to the data points. Position of the peak (centered at $D_{L}=3.7 \times 10^{-7} \mathrm{~cm}^{2} \mathrm{~s}^{-1}$ ) is set as $D_{L 0}$ for the following normalization of $D_{L}$ values in other systems. 


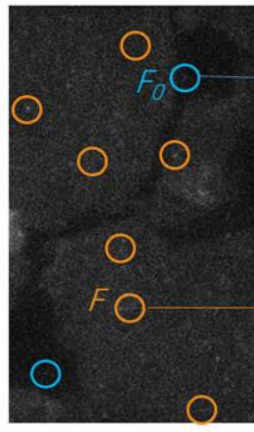

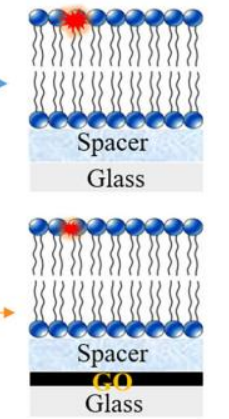

$\mathrm{b}$

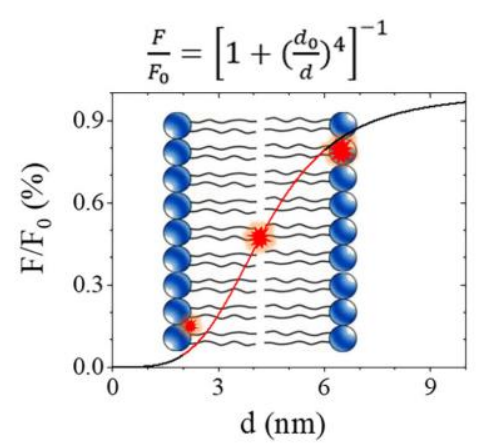

c

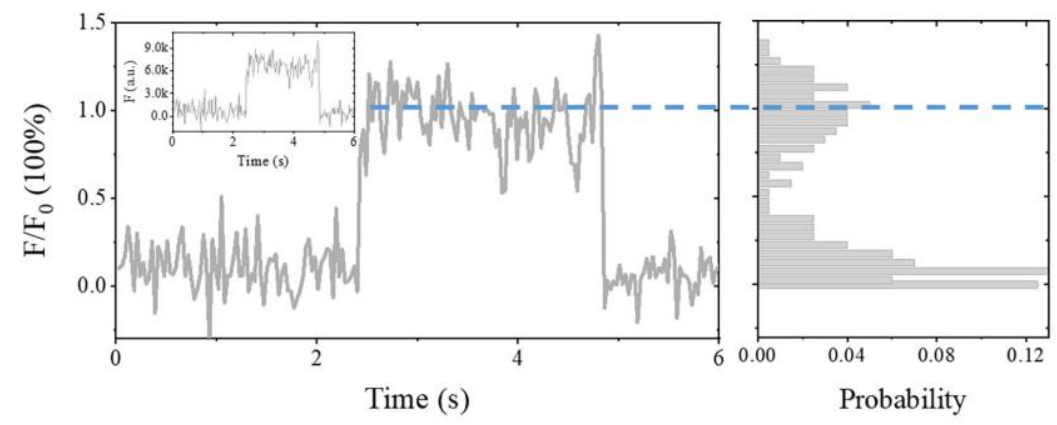

d
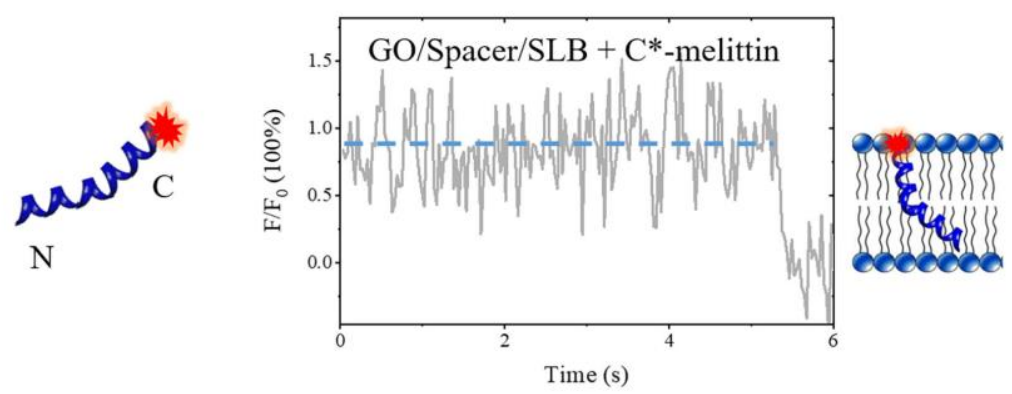

Figure S9. SIFA tests. (a) Setup of the SIFA tests of RhB-labeled DOPC bilayer supported on a glass/GO/PEG substrate. The fluorescence intensity of molecules on the GO area (grey region) was taken as $F$ while that of molecules on a glass/PEG (without GO) region was taken as $F_{0}$ for reference. The image refers to an actual area of $28 \times 48 \mu \mathrm{m}$. (b) Quantitative relationship between the normalized fluorescence intensity $F / F_{0}$ and z-position of fluorophores. (c) Typical fluorescence trace of a RhB-lipid before (shown in the inset) or after fluorescence intensity normalization. Corresponding statistical distributions of the dynamic fluorescence intensities weighted with their dwell times are shown on the right. (d) Typical fluorescence trace of C-terminal labelled melittin on a GO-supported bilayer.

\section{Corresponding Notes}

In the SIFA experiment, the fluorescent dye works as an energy donor and a graphene oxide 
(GO) monolayer works as an energy acceptor. Based on this, changes in distance from the dye to the GO plane would be directly reflected as changes in fluorescence intensity of the dye in real time. Here, a GO monolayer is selected as its effective quenching range (i.e., $1 \sim 9 \mathrm{~nm}$ ) best fit the thickness of a cell membrane (Figure S7b). ${ }^{7}$ Therefore, the GO-based SIFA technique provides a useful method to monitor the vertical translocation of individual lipid or peptide molecules in relative to a cell membrane, at a sub-nanometer level.

In our experiments, a similar supported DOPC bilayer on a graphene oxide (and a polyethylene glycol layer as a spacer)-coated glass substrate (Figure S7a) was incubated with melittin at different concentrations (i.e., $0,0.5$ and $5.0 \mu \mathrm{g} \mathrm{mL}^{-1}$ ) for $30 \mathrm{~min}$ before video capture. Here, the graphene oxide monolayer works as an energy acceptor plane, and the actual fluorescence intensity of dye (refers to the dye-labeled lipid or peptide) is influenced by the distance from the dye to such GO plane. The changes in fluorescence intensity of individual lipids with time $(\mathrm{F} \sim \mathrm{t}$; at a $30 \mathrm{~ms}$ time interval) were obtained thereafter, which were then normalized with the fluorescence intensity of lipids on the substrate region without $\mathrm{GO}$ (as the pristine fluorescence intensity of a free dye, $\mathrm{F}_{0}$ ) and further transferred to changes in z-position (from $F / F_{0} \sim \mathrm{t}$ to $\mathrm{d} \sim \mathrm{t}$, Figure $\mathrm{S} 7 \mathrm{~b}$ ).

a

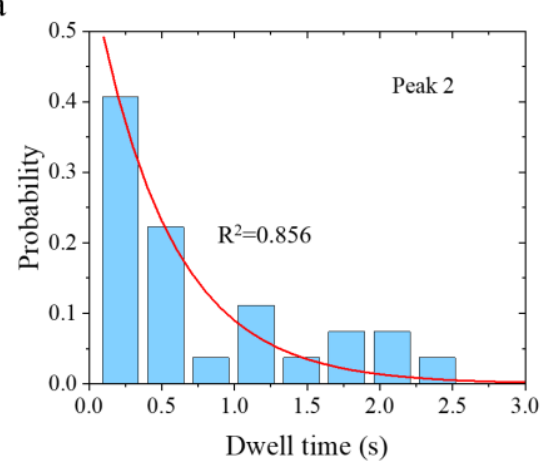

b

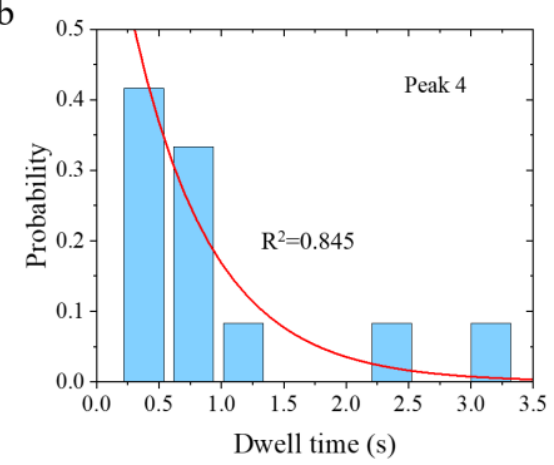

Figure S10. PDF distribution of dwell time referring to peak 2 and peak 4 in Figure $4 \mathrm{c}$ in the main text. An exponential function $y=a * \exp (b * x)$ was used to fit the distribution and corresponding parameters were shown in Table S3. 


\section{Supplementary tables}

Table S1. Gaussian and exponential fittings of the normalized displacement distributions, i.e., P $\left(\Delta \mathrm{x}^{\prime}\right)$ and $\mathrm{P}\left(\Delta \mathrm{y}^{\prime}\right)$, in different systems as shown in Figure 1c in the main text. Confidence intervals (95\%) of the fits were also shown in the table. The result of testing goodness of fit was shown in

Table S4.

\begin{tabular}{|c|c|c|c|c|c|}
\hline \multirow{2}{*}{ Sample } & \multicolumn{2}{|c|}{ Gaussian (Black profile) } & \multicolumn{2}{c|}{ Exponential (Red profile) } \\
& \multicolumn{2}{|c|}{$y=a \exp \left(-1 / 2((x-b) / c)^{\wedge} 2\right)$} & \multicolumn{2}{c|}{$y=a \exp (-b|x|)$} \\
\hline Pristine & a & b & $\mathrm{c}$ & $\mathrm{a}$ & $\mathrm{b}$ \\
\hline $0.5 \mu \mathrm{g} \mathrm{mL}^{-1}$ & $0.085 \pm 0.002$ & $0.011 \pm 0.021$ & $0.886 \pm 0.021$ & $0.558 \pm 0.002$ & $2.005 \pm 0.030$ \\
\hline $5.0 \mu \mathrm{g} \mathrm{mL}^{-1}$ & $0.082 \pm 0.002$ & $0.016 \pm 0.024$ & $0.903 \pm 0.024$ & $0.234 \pm 0.002$ & $1.504 \pm 0.035$ \\
\hline
\end{tabular}

Table S2. Gaussian fittings of the PDF histogram of diffusion coefficient, i.e. P $\left(D_{L}\right)$, of mobile lipids based on time-averaged MSD of an overall of 400 representative lipid trajectories as shown in Figure 1d in the main text. The $D_{L}$ values were then normalized with that of lipids in a pristine membrane without Mel exposure. The result of testing goodness of fit was shown in Table S6.

Before Normalization $\left(\mu \mathrm{m}^{2} \mathrm{~s}^{-1}\right)$

\begin{tabular}{|c|c|c|c|c|c|c|}
\hline \multirow{2}{*}{ Sample } & \multicolumn{2}{|c|}{ Peak 1 (in Green) } & \multicolumn{2}{|c|}{ Peak 2 (in Blue) } & \multicolumn{2}{|c|}{ Peak 3 (in Red) } \\
\cline { 2 - 7 } & Center & Area & Center & Area & Center & Area \\
\hline Pristine & - & - & 2.780 & $0.479(100 \%)$ & - & - \\
\hline $0.5 \mu \mathrm{g} \mathrm{mL}^{-1}$ & 1.569 & $0.196(42.6 \%)$ & 2.842 & $0.185(40.2 \%)$ & 4.383 & $0.079(17.2 \%)$ \\
\hline $5.0 \mu \mathrm{g} \mathrm{mL}^{-1}$ & 1.534 & $0.366(71.3 \%)$ & 2.769 & $0.147(28.7 \%)$ & - & - \\
\hline
\end{tabular}

After Normalization

\begin{tabular}{|c|c|c|c|c|c|c|}
\hline \multirow{2}{*}{ Sample } & \multicolumn{2}{|c|}{ Peak 1 (in Green) } & \multicolumn{2}{|c|}{ Peak 2 (in Blue) } & \multicolumn{2}{|c|}{ Peak 3 (in Red) } \\
\cline { 2 - 7 } & Center & Area & Center & Area & Center & Area \\
\hline Pristine & - & - & 1.0 & $0.5(100 \%)$ & - & - \\
\hline $0.5 \mu \mathrm{g} \mathrm{mL}^{-1}$ & 0.6 & $0.2(42.6 \%)$ & 1.0 & $0.2(40.2 \%)$ & 1.6 & $0.1(17.2 \%)$ \\
\hline $5.0 \mu \mathrm{g} \mathrm{mL}^{-1}$ & 0.6 & $0.4(71.3 \%)$ & 1.0 & $0.1(28.7 \%)$ & - & - \\
\hline
\end{tabular}


Table S3. Exponential fittings, with $y=a * \exp (b * x)$, of the dwell time distributions referring to peak 2 and peak 4 in Figure 4c, as shown in Figure S10. Confidence intervals of the fits were also shown in the table.

\begin{tabular}{|c|c|c|}
\hline & a & b \\
\hline Peak 2 & $0.594 \pm 0.283$ & $-1.891 \pm 1.099$ \\
\hline Peak 4 & $0.803 \pm 0.525$ & $-1.562 \pm 1.060$ \\
\hline
\end{tabular}




\section{Data analysis details}

Table S4. Goodness of fit test of the Gaussian fittings in Figure 1c

Function $\mathrm{y}=a * \exp \left(-1 / 2((x-b) / c)^{\wedge} 2\right)$

\begin{tabular}{ccc}
\hline Sample & $\mathrm{R}^{2}$ & SSE \\
\hline Pristine & 0.968 & 0.008 \\
\hline $0.5 \mu \mathrm{g} \mathrm{mL}^{-1}$ & 0.977 & 0.005 \\
\hline $5.0 \mu \mathrm{g} \mathrm{mL}^{-1}$ & 0.959 & 0.009 \\
\hline
\end{tabular}

Table S5. Goodness of fit test of Figure 1d

Function $\mathrm{y}=\sum\left(a * \exp \left(-1 / 2((x-b) / c)^{\wedge} 2\right)\right)$

\begin{tabular}{cccc}
\hline Sample & $\mathrm{R}^{2}$ & Fit Std Error & F-value \\
\hline Pristine & 0.957 & 0.015 & 154.870 \\
\hline $0.5 \mu \mathrm{g} \mathrm{mL}^{-1}$ & 0.937 & 0.017 & 27.083 \\
\hline $5.0 \mu \mathrm{g} \mathrm{mL}^{-1}$ & 0.841 & 0.036 & 15.891 \\
\hline
\end{tabular}

Table S6. Goodness of fit test of Figure 2d

\begin{tabular}{ccc}
\hline $\mathrm{R}^{2}$ & Fit Std Error & F-value \\
\hline 0.817 & 0.016 & 17.131 \\
\hline
\end{tabular}

Table S7. Goodness of fit test of Figure 3b

\begin{tabular}{ccc}
\hline $\mathrm{R}^{2}$ & Fit Std Error & F-value \\
\hline 0.984 & 0.007 & 113.843 \\
\hline
\end{tabular}

Table S8. Goodness of fit test of Figure 4c

\begin{tabular}{ccc}
\hline $\mathrm{R}^{2}$ & Fit Std Error & F-value \\
\hline 0.907 & 0.015 & 28.354 \\
\hline
\end{tabular}




\section{Supplementary equations and corresponding notes}

The radius of gyration $\mathrm{Rg}$ was calculated as

$R_{g}^{2}=\frac{1}{N} \sum_{i=1}^{N}\left[\left(x_{i}-\langle x\rangle\right)^{2}+\left(y_{i}-\langle y\rangle\right)^{2}\right]$

and normalized as

$R_{g}^{\prime}=R_{g} /\left\langle R_{g}^{2}\right\rangle^{1 / 2}$

The ensemble-averaged MSD $\left\langle\Delta \vec{r}^{2}(\tau)\right\rangle$ of each system was calculated according to the function of

$$
\operatorname{MSD}=\frac{1}{N} \sum_{1}^{N}(\vec{r}(\tau)-\vec{r}(0))^{2}
$$

\section{Notes}

Here, the ensemble-averaged MSD of each systems was calculated as a function of $\tau$ and displayed in Figure S4a. The black and red circles were obtained from all lipid trajectories or after removing the immobile ones, respectively. It is observed that the profiles from the three systems demonstrate similar $\tau$-dependent trend. By piecewise fitting, the small time-lag range of $\tau<0.2 \mathrm{~s}$ exhibits an anomalous diffusive behavior with $\left\langle\Delta \vec{r}^{2}(\tau)\right\rangle \sim \tau^{\alpha}$, where $\alpha \approx 0.90 \pm 0.04<1$. Such sub-diffusion is normally observed in a variety of dense fluid or cell membrane systems. ${ }^{8}$ In contrast, in the longtime diffusion regime $(\tau>0.2 \mathrm{~s})$, the MSD demonstrates a linear dependence of $\tau$ with $\left\langle\Delta \vec{r}^{2}(\tau)\right\rangle \simeq$ $4 D_{L} \tau$, where $D_{L}$ stands for the long-time diffusion constant. Such linear dependence indicates that the membrane is fluidic for lipid diffusion.

The time-averaged MSD based on the trajectory of single particles in terms of the time series $\vec{r}\left(t^{\prime}\right)$ over a total measurement time $t$, was calculated with

$\left\langle\Delta \vec{r}^{2}(\tau)\right\rangle=\frac{1}{t-\tau} \int_{0}^{t-\tau}\left(\vec{r}\left(t^{\prime}+\tau\right)-\vec{r}\left(t^{\prime}\right)\right)^{2} d t^{\prime}$

as a function of lag time $\tau$ much shorter than t. Representative MSD profiles of lipids in the three conditions were displayed in Figure S4b. 


\section{Experimental details}

\section{Materials}

All types of phospholipids, such as 1,2-dioleoyl-sn-glycero-3-phosphocholine (DOPC), 1,2dioleoyl-sn-glycero-3-phosphoethanolamine-N-(lissamine rhodamine B sulfonyl (RhB-PE), 1palmitoyl-2-(6-[(7-nitro-2-1,3-benzoxadiazol-4-yl)amino]hexanoyl)-sn-glycero-3phosphoethanolamine (16:0-06:0 NBD-PE), were purchased from Avanti Polar Lipids. Calcein, graphene oxide dispersion, 1-Aminopyrene, hydroxyl-PEG-NHS and chloroform were purchased from Sigma Aldrich.

\section{GUV leakage assay}

GUVs (DOPC labeled with $0.5 \mathrm{~mol} \% \mathrm{Rh}-\mathrm{PE}$ ), with the encapsulated calcein, were prepared following the conventional electroformation method..$^{9-10}$ In brief, a solution of DOPC/Rh-PE lipids $\left(60 \mu \mathrm{L} \times 2.0 \mathrm{mg} \mathrm{mL}^{-1}\right.$ in chloroform) was deposited onto two ITO-coated glass slides and dried under vacuum overnight. The dry film was transferred in a homemade electro-formation chamber (with the two glass slides as electrodes), and rehydrated in $0.1 \mathrm{M}$ sucrose buffer containing $0.2 \mathrm{mg}$ $\mathrm{mL}^{-1}$ calcein. Alternating voltages were applied $(0.5 \mathrm{~V} \times 20 \mathrm{~min}, 1.0 \mathrm{~V} \times 20 \mathrm{~min}$ and $1.5 \mathrm{~V} \times 3 \mathrm{~h})$. The obtained vesicles were washed three times via centrifugation $(8000 \mathrm{rpm} \times 20 \mathrm{~min})$. Well dispersed GUVs with a size distribution of 8-30 $\mu \mathrm{m}$ and encapsulated calcein were collected and redispersed in $0.1 \mathrm{M}$ glucose solution $\left(\sim 0.02 \mathrm{mg}\right.$ lipid $\left.\mathrm{mL}^{-1}\right)$ for use.

A volume of $500 \mu \mathrm{L}$ of the GUV dispersion was moved to a home-made cell for an in-situ observation under confocal microscope (LSM 710, Zeiss) equipped with a 100× oil objective. RhBlabeled lipids were excited by a HeNe laser (EX $543 \mathrm{~nm}$ ) and the fluorescence was observed through filter set 20 (EM BP 575-640 nm). Calcein were excited by an argon ion laser (EX $488 \mathrm{~nm}, 25 \mathrm{~mW}$ ) and their fluorescence was observed through filter set 44 (EM BP 530/50 nm). The data was acquired with a pixel dwell time of $1.58 \mu$ s and it generally took $3.87 \mathrm{~s}$ to take one image. The transmission channel illuminated with a Halogen lamp was acquired meanwhile. After stabilization of the GUVs on the substrate, $100 \mu \mathrm{L}$ of melittin solution was added gently, to a final peptide concentration of $9.0,8.0, \ldots, 3.0,2.5,2.0,1.5,1.0,0.5 \mu \mathrm{g} / \mathrm{mL}$, respectively. Peptide-induced content-release and deformation of the GUVs were monitored in real time. All images were captured 
under the same instrumental settings.

\section{Single molecular tracking}

Experimental setup. SLB (DOPC containing $0.01 \mathrm{~mol} \%$ fluorescence-labeled PE) was deposited on the glass substrate of a home-made cell by the traditional vesicle fusion method. ${ }^{11-12}$ In brief, a solution of monodisperse small unilamellar lipid vesicles (SUVs), with a typical size of $110 \pm 15$ $\mathrm{nm}$, was firstly prepared by the conventional extrusion method. $50 \mu \mathrm{L}$ of the SUV dispersion was moved to the home-made cell with an Ismatec peristaltic pump (Sweden) at a fixed liquid rate at 50 $\mu \mathrm{L} \mathrm{min}{ }^{-1}$, and incubated at room temperature for $2 \mathrm{~h}$, during which SLB with high quality was formed through vesicle fusion. After that, the cell was gently washed with DI water to remove the excessive lipids.

The SLB was monitored under a microscope (Olympus, IX71) with a TIRF-specialized 100× oil objective (Olympus, UPLAPO100XOHR) and an Electron-Multiplying CCD (Andor, DU$897 \mathrm{U}$ ). A $50 \mu \mathrm{L}$ solution of melittin (at $0,0.5$ or $5.0 \mu \mathrm{g} / \mathrm{mL}$ concentration) was pumped into the cell at $50 \mu \mathrm{L} \mathrm{min}{ }^{-1}$ and incubated for $30 \mathrm{~min}$. After that, fluorescence image or video (30 fps $\times 18 \mathrm{~s}$ ) was captured in situ. The RhB fluorophore was excited by a Sapphire $532 \mathrm{~nm}$ laser (Coherent; at $60 \%$ of the $200 \mathrm{~mW}$ power) equipped with an optical splitter (Andor, OptoSplitII, FF560-FDi01, 25 $\times 36 \mathrm{~mm}$ in size). The fluorescence was collected through a filter FF01 (EM BP 585/40, $25 \mathrm{~mm}$ in size) and an optical splitter (Andor, OptoSplitII, FF640-FDi01, 25×36 mm in size).

Data analysis was carried out following a previous method. ${ }^{13}$ In short, the images were first filtered using a Gaussian kernel with a standard deviation of 1.0 pixel in ImageJ. After that, the 2D single particle tracking module in Mosaic of ImageJ was used to obtain the molecular trajectories.

Corresponding Note. An incubation time of $30 \mathrm{~min}$ was selected before microscope observation in the experiments. Both our GUV leakage and QCM-D experiments show that, at various peptide concentrations (from 2.5 to $8.0 \mu \mathrm{g} \mathrm{mL}^{-1}$ ) the melittin-membrane interactions occur within $20 \mathrm{~min}$ after peptide addition..$^{3,11-12}$ Therefore, after $30 \mathrm{~min}$ incubation, an equilibrium state of membrane (with the adsorbed or inserted peptides) should have been reached. 


\section{SIFA tests}

Experimental setup. The SIFA tests were carried out following the previous operations. ${ }^{14}$ In brief, a monolayer of graphene oxide (GO) sheets was firstly deposited on a pre-cleaned glass surface by the Langmuir-Blodgett method (KSV NIMA, Biolin). A volume of $500 \mu \mathrm{L}$ 1-Aminopyrene (at 1 $\mathrm{mM}$ ) and hydroxyl-PEG5k-NHS (at $1 \mathrm{mM}$ ) was used to incubate the substrate for $30 \mathrm{~min}$, and then washed carefully with DI water. A layer of PEG molecule $(1.3 \pm 0.2 \mathrm{~nm}$ in thickness) would be deposited on the GO surface as a spacer. A DOPC bilayer (containing $0.01 \%$ headgroup- or taillabeled RhB- and/or NBD-PE) was then deposited on the PEG-coated GO substrate by the vesicle fusion method. The membrane was then incubated with melittin at different concentrations (i.e., 0 , 0.5 and $5.0 \mu \mathrm{g} \mathrm{mL}^{-1}$ ) for 30 min before video capture under the TIRF microscope. For peptide monitoring in the SIFA experiments, pristine lipids without fluorescent labeling were used for bilayer preparation, while $1 \mathrm{~mol} \% \mathrm{RhB}$-labeled melittin (on the $\mathrm{N}$ - or C-terminal) was mixed with pristine melittin for membrane incubation.

Data collection. The fluorescence intensity value of molecules on the glass/GO/PEG substrate (i.e., $F$ ) was normalized with that of molecules on the glass/PEG (without $\mathrm{GO}$ ) region (i.e., $F_{0}$ ) in the same specimen (Figure $\mathrm{S} 7 \mathrm{a}$ ). The as acquired $F / F_{0}$ can be transferred to the distance from the GO surface (i.e., $d$ ) and therefore z-position of the molecule in relative to the bilayer would be obtained (Figure S7b).

Step fitting algorithm. The step fits in Figure 3 and 4 (red lines) were produced following a previous step-fitting algorithm as detailed in Nature 2006, 442 (7103), 709. ${ }^{15}$ In brief, two steps were carried out. (1) Finding steps. Based on a calculation of the Chi-square, we firstly choose a single large step to fit the data. Subsequent steps are obtained through fitting new steps to the plateaus of previous ones. Each time, the most prominent one is selected. Eventually, a series of "best fits" with only one-step difference are obtained. (2) Evaluating the quality. A "counter fit" of the original "best" fit is used, which has the same number of steps as the original fit but with step locations in between the step locations. Each "best" fit in the series is compared to such "counter" fit, with a 'step-indicator' defined as the ratio between the Chi-square of the counter fit and the Chisquare of the best fit. When the number of steps in the best fit is very close to the real number of steps in the data, the value of the "step indicator" will be large. 
Corresponding Note. Surface-induced fluorescence attenuation (SIFA) technique ${ }^{7}$ was used to track the vertical movement of individual fluorophore-labeled lipids or peptides in real time, as the fluctuations in fluorescence intensity $\mathrm{F}$ of the fluorophore directly reflects the changes in $\mathrm{z}$ position $d$ of it from the quenching plane of the substrate graphene oxide (GO) layer as

$\frac{F}{F_{0}}=\left[1+\left(\frac{d_{0}}{d}\right)^{4}\right]^{-1}$

where $F_{0}$ and $d_{0}$ refer to the fluorescence intensity of a free fluorophore and the characteristic quenching distance at which $F / F_{0}=1 / 2$. According to previous reports, the characteristic quenching distance is derived to be $\mathrm{d}_{0}=4.0 \pm 0.5 \mathrm{~nm}$ for the $\mathrm{GO}$ monolayers used.

\section{MD simulations}

All simulations are performed using GROMACS 5.1.4 with the MARTINI 2.2 force field. ${ }^{16-17}$ The isothermal-isobaric (NPT) ensemble is applied in the simulations with a temperature of $310 \mathrm{~K}$ and pressure of 1.0 bar. The temperature and pressure are controlled with the Berendsen temperature coupling scheme with a time constant of $1 \mathrm{ps}$ and the Berendsen's pressure scaling method with a time constant of $12 \mathrm{ps}$ and a compressibility of $3 \times 10^{-4} \mathrm{bar}^{-1},{ }^{18}$ respectively. Non-bonded interactions, including electrostatics and Lennard-Jones, are cut off at $1.1 \mathrm{~nm}$. Periodic boundary conditions are applied in all directions, and the time step is set to be $20 \mathrm{fs}$.

The lipid bilayer membrane used in the simulations is set to be parallel to the $x-y$ plane of the simulation box and consists of 1058 DLPC lipid molecules. The coarse-grained (CG) model of Mel is obtained by using the Martinize script with the crystal structure of peptide (PDB ID: 2MLT). In our simulations, the peptide number is determined by the Peptide-to-lipid ratio (P/L). In addition, $0.1 \mathrm{M} \mathrm{NaCl}$ is included in the system and additional $\mathrm{Cl}^{-}$ions are added to neutralize the charge on the peptides. The total simulation time is at least $T=10,000 \mathrm{~ns}$.

To calculate the local diffusion of lipids, the lipids in each leaflet are split into grids, each one with area of $1 \mathrm{~nm}^{2}$. Then the local mean squared displacement is calculated as $\operatorname{Local} M S D(t)=$ $\frac{1}{N_{l}} \sum_{n_{l}=0}^{N_{l}-1}\left[\boldsymbol{r}\left(\left(t+n_{l}\right)+t_{0}\right)-\boldsymbol{r}(t)\right]^{2}$ in which $t_{0}=1 n s, N_{l}=10 .{ }^{19}$ To avoid the high fluctuation of lipid distribution in each grid and obtain a statistically meaningful contour map, Local MSD is 
averaged with $N_{l}$ consecutive contour maps at times $t, t+1, \ldots, t+\left(N_{l}-1\right) n s$.

\section{Reference}

1. Huang, H. W. Molecular Mechanism of Antimicrobial Peptides: The Origin of Cooperativity. BBABiomembranes 2006, 1758 (9), 1292-1302.

2. Santo, K. P.; Berkowitz, M. L. Difference between Magainin-2 and Melittin Assemblies in Phosphatidylcholine Bilayers: Results from Coarse-Grained Simulations. J Phys Chem B 2012, 116 (9), 3021-3030.

3. Liu, J. J.; Xiao, S. F.; Li, J. L.; Yuan, B.; Yang, K.; Ma, Y. Q. Molecular Details on the Intermediate States of Melittin Action on a Cell Membrane. BBA-Biomembranes 2018, 1860 (11), 2234-2241.

4. Deng, Z. X.; Li, J. L.; Yuan, B.; Yang, K. Residue-Specialized Membrane Poration Kinetics of Melittin and Its Variants: Insight from Mechanistic Landscapes. Commun Theor Phys 2019, 71 (7), 887902.

5. Woo, S. Y.; Lee, H. Aggregation and Insertion of Melittin and Its Analogue MelP5 into Lipid Bilayers at Different Concentrations: Effects on Pore Size, Bilayer Thickness and Dynamics. Phys Chem Chem Phys 2017, 19 (10), 7195-7203.

6. Krapf, D.; Campagnola, G.; Nepal, K.; Peersen, O. B. Strange Kinetics of Bulk-Mediated Diffusion on Lipid Bilayers. Phys Chem Chem Phys 2016, 18 (18), 12633-12641.

7. Li, Y.; Qian, Z. Y.; Ma, L.; Hu, S. X.; Nong, D. G.; Xu, C. H.; Ye, F. F.; Lu, Y.; Wei, G. H.; Li, M. Single-Molecule Visualization of Dynamic Transitions of Pore-Forming Peptides among Multiple Transmembrane Positions. Nat Commun 2016, 7, 12906.

8. Aspelmeyer, M.; Meystre, P.; Schwab, K. Quantum Optomechanics. Phys Today 2012, 65 (7), 2935.

9. Xiao, S. F.; Lu, X. M.; Gou, L.; Li, J. L.; Ma, Y. Q.; Liu, J. J.; Yang, K.; Yuan, B. Graphene Oxide as Antibacterial Sensitizer: Mechanically Disturbed Cell Membrane for Enhanced Poration Efficiency of Melittin. Carbon 2019, 149, 248-256.

10. Lu, X. M.; Liu, J. J.; Gou, L.; Li, J. L.; Yuan, B.; Yang, K.; Ma, Y. Q. Designing Melittin-Graphene Hybrid Complexes for Enhanced Antibacterial Activity. Adv Healthc Mater 2019, 8 (9), e1801521.

11. Lu, N. Y.; Yang, K.; Li, J. L.; Yuan, B.; Ma, Y. Q. Vesicle Deposition and Subsequent MembraneMelittin Interactions on Different Substrates: A QCM-D Experiment. BBA-Biomembranes 2013, 1828 (8), 1918-1925.

12. Lu, N. Y.; Yang, K.; Yuan, B.; Ma, Y. Q. Molecular Response and Cooperative Behavior during the Interactions of Melittin with a Membrane: Dissipative Quartz Crystal Microbalance Experiments and Simulations. J Phys Chem B 2012, 116 (31), 9432-9438.

13. Sbalzarini, I. F.; Koumoutsakos, P. Feature Point Tracking and Trajectory Analysis for Video Imaging in Cell Biology. J Struct Biol 2005, 151 (2), 182-195.

14. Ma, L.; Hu, S. X.; He, X. L.; Yang, N.; Chen, L. C.; Yang, C. G.; Ye, F. F.; Wei, T. T.; Li, M. Detection of tBid Oligomerization and Membrane Permeabilization by Graphene-Based SingleMolecule Surface-Induced Fluorescence Attenuation. Nano Lett 2019, 19 (10), 6937-6944.

15. Kerssemakers, J. W. J.; Munteanu, E. L.; Laan, L.; Noetzel, T. L.; Janson, M. E.; Dogterom, M. Assembly Dynamics of Microtubules at Molecular Resolution. Nature 2006, 442 (7103), 709-712. 
16. Marrink, S. J.; Risselada, H. J.; Yefimov, S.; Tieleman, D. P.; de Vries, A. H. The MARTINI Force Field: Coarse Grained Model for Biomolecular Simulations. J Phys Chem B 2007, 111 (27), 7812-7824. 17. Pronk, S.; Pall, S.; Schulz, R.; Larsson, P.; Bjelkmar, P.; Apostolov, R.; Shirts, M. R.; Smith, J. C.; Kasson, P. M.; van der Spoel, D.; Hess, B.; Lindahl, E. GROMACS 4.5: a High-Throughput and Highly Parallel Open Source Molecular Simulation Toolkit. Bioinformatics 2013, 29 (7), 845-854.

18. Berendsen, H. J. C.; Postma, J. P. M.; Vangunsteren, W. F.; Dinola, A.; Haak, J. R. MolecularDynamics with Coupling to an External Bath. J Chem Phys 1984, 81 (8), 3684-3690.

19. Jeon, J. H.; Javanainen, M.; Martinez-Seara, H.; Metzler, R.; Vattulainen, I. Protein Crowding in Lipid Bilayers Gives Rise to Non-Gaussian Anomalous Lateral Diffusion of Phospholipids and Proteins. Phys Rev X 2016, 6 (2), 021006. 